Research Article

\title{
Tangential Change Behavior and Pedestrian Simulation of Multichannel Evacuation Crowd
}

\author{
Lianghai Jin, ${ }^{1,2,3,4}$ Mei Fang $\mathbb{D}^{1,2}$ Shu Chen, ${ }^{1,2}$ Wenfan Lei, ${ }^{1,2}$ and Yun Chen ${ }^{1,2}$ \\ ${ }^{1}$ Key Laboratory of Construction and Management in Hydropower Engineering, China Three Gorges University, \\ Yichang 443002, Hubei, China \\ ${ }^{2}$ College of Hydraulic \& Environmental Engineering, China Three Gorges University, Yichang 443002, Hubei, China \\ ${ }^{3}$ Safe-Environment Technology Co., Ltd., Three Gorges University, Yichang 443002, Hubei, China \\ ${ }^{4}$ Center of Standardization Evaluation for Production Safety, China Three Gorges University, Yichang 443002, Hubei, China
}

Correspondence should be addressed to Mei Fang; 201908710021011@ctgu.edu.cn

Received 10 July 2020; Revised 13 August 2020; Accepted 1 October 2020; Published 22 October 2020

Academic Editor: Anna M. Gil-Lafuente

Copyright (C) 2020 Lianghai Jin et al. This is an open access article distributed under the Creative Commons Attribution License, which permits unrestricted use, distribution, and reproduction in any medium, provided the original work is properly cited.

In evacuation, the velocity difference of adjacent pedestrians before and after often leads to tangential change of pedestrian location in channel. This tangential change behavior and its interaction disturb the stable state of crowd evacuation in multiple channels, which can affect the efficiency of crowd evacuation and even cause trampling accidents. This paper considers the dynamic comfort distance and the expected speed and analyzes the relative position changes after pedestrians change lanes. It investigates the conditions of tangential change behavior and defines the rules of tangential change behavior processing. Meanwhile, it investigates the crowd's tangential change behavior and its interaction process, revealing the crowd evacuation mechanism of tangential change behavior conditions. Simulation results show that as the crowd density gradually increases, pedestrians exhibit the evolutionary characteristic of "no tangential change $\longrightarrow$ occasional tangential change $\longrightarrow$ frequent tangential change $\longrightarrow$ closely following." The evacuation speed is obviously influenced by pedestrian's tangential change behavior and crowd density; when the pedestrian density $\rho=2.0$ and $\rho=3.0$, the tangential change behavior not only makes the speed difference and fluctuation between different lanes great but also has the same effect on the average speed of pedestrians. The results of this study can provide theoretical insights into the organization of multichannel evacuation and expand the theoretical space of crowd dynamics in an evacuation.

\section{Introduction}

Trampling accidents of crowded place from time to time such as tourism blocks, school building passages, movie theaters, and such crowd management problems have become a hotspot for research on evacuation behavior [1]. The unbalanced initial location distribution and complex and variable motion characteristics of the evacuated crowd result in a speed difference between the front and adjacent pedestrians [2]. Besides, there is subjective variation in the expected speed of pedestrians, with some pedestrians intending to overtake the immediate predecessor pedestrians and change lanes to adjacent lanes, resulting in tangential change behavior. This behavior interacts with other pedestrians, thus disturbing the orderly state of multistreet crowd evacuation which gradually shifts to a chaotic state. Therefore, it is of considerable significance to study the characteristics of the tangential change behavior of the crowd and the passage law of the multichannel road evacuation in order to evacuate the crowd quickly and efficiently.

For the study of the causation mechanism of pedestrian evacuation process, most scholars believe that crowd hedging and surging, pedestrian competition, and coaxing are critical influences of crowd trampling accidents [3]. In addition, the trajectory and path selection of pedestrians has an important influence on the speed and flow of crowd evacuation [4]. Hua et al. [5] argued that abnormal 
behaviors such as crowding, fleeing, congregating, and retrograde behavior in public places tended to be more harmful. Especially in high-density crowds, pedestrians' comfort distance is squeezed and pedestrians develop anxiety and irritation, making dense crowds highly susceptible to trampling accidents at bottlenecks such as escalators and exits, resulting in casualties [6]. It can be seen that the dense crowd with undesirable interaction behaviors are the key causes of pedestrian accidents. Therefore, Huang et al. [7] proposed that focusing on the behavioral characteristics of pedestrians in crowd hedging, anisotropic flow, and unidirectional flow and analyzing the abnormal behaviors therein are the theoretical basis for reducing the risk of crowd trampling and improving evacuation efficiency. Moussaid et al. [8] had organized a two-way flow loop experiment where pedestrians were allowed to overtake other pedestrians through a tangent and found that the variability in pedestrian speed was the main cause of the instability in pedestrian traffic flow. Yuen and Lee [9] found that faster walking pedestrians tend to overtake slower walking pedestrians in unidirectional pedestrian flows. Heliövaara et al. [10] argued that pedestrians can interact autonomously based on their surroundings by adjusting their walking route accordingly to avoid conflicts with front pedestrians as much as possible. The aforementioned studies illustrate that pedestrian tangential change behavior is usually accompanied by overtaking behavior and route choice behavior. Compared with previous studies, they have paid more attention to the pedestrian's overtaking behavior and route choice behavior under limited vision $[11,12]$. Although the studies reflect the role of partial shear behavior, there is less analysis of the mechanism of the effects of pedestrian tangential change.

This paper combines the perceived comfort distance and pedestrian density and considers the different desired speeds between pedestrians that lead to pedestrian occurrence tangential behavior. Tolerance (i.e., the amount of time a pedestrian can tolerate forced deceleration) is then proposed as the adjudicative value of the pedestrian's choice of tangential change, with an analysis of subjective pedestrian influences on the occurrence of tangential behavior. Therefore, this paper focuses on the perturbation of pedestrian tangential change behavior in multilane evacuation. It analyzes the characteristics and interaction process of pedestrian shear behavior, investigates the conditions under which pedestrian shear behavior occurs, and determines the rules of pedestrian movement and conflict handling. Besides, it constructs an evacuation crowd passage model for multiple lanes under tangential change behavior and conducts simulations using a cellular automaton method to reveal the influence mechanism of tangential change behavior on the efficiency of multilane evacuation pedestrian passage.

To sum up, the innovation point of this paper is, at the micro level of tangential change behavior, loading the minimum demand distance and tolerance variables and using the simulation method to explain the macroscopic law of multichannel pedestrian passage.

\section{Tangential Change Behavior of Evacuating Crowds in Multiple Channels}

2.1. Interaction Characteristics and Processes. In public buildings, with the interactions among pedestrians and the uncertainty of pedestrian density, pedestrian traffic is a nonlinear dynamic stochastic problem [13]. Pedestrians on foot choose to move forward and backward, wait, move left and right, exchange positions, and show other behaviors based primarily on their surroundings, and the actual situation within their field of vision determines their behavioral choices [14]. The space reserved by pedestrian walking should not only meet the need for space to swing sideways but also maintain a comfort or safety distance [15], which can be referred to as personal comfort distance. On the one hand, personal comfort distance varies across pedestrians as it is related to their walking speed and reaction speed. On the other hand, the habit of following results in approximately the same speed of passage for pedestrian immediately in front and behind. Thus, the pedestrian interaction process is dynamically stochastic. As shown in Figure 1, pedestrians on the track will adjust the walking distance between them and the pedestrians immediately ahead and behind them according to their speed at any time. In this paper, the distance maintained by the pedestrian from the immediate front walker is referred to as the immediate front comfort distance (the direct predecessor comfort distance is the same as the immediate front comfort distance). In the figure, $L^{c}$ indicates the immediate front comfort distance and $L^{a}$ indicates the actual distance between the pedestrian and the immediate front walker.

In reality, pedestrians are different individuals with heterogeneity [16], so the pedestrian actual distance with the immediate predecessor pedestrian should be greater than or equal to his comfort distance. When a pedestrian is following the immediate predecessor, the primary consideration is that when the pedestrian suddenly stops or slows down, he has sufficient distance to react. Thus, the actual distance $L^{a}$ can be expressed as the sum of the response distance and the minimum demand distance. Combining the traffic characteristics of the passage and the staircase, we introduce the horizontal angle $\theta$; when $\theta=0$, it represents the horizontal passage area. When $\theta \neq 0$, it indicates nonhorizontal access areas like stairs. The actual demand distance $L^{a}$ can be expressed as

$$
L^{a}=t_{r} V_{f} \cos \theta+l_{\min }
$$

In (1), $t_{r}$ means response time, $V_{f}$ means pedestrian speed, and $l_{\text {min }}$ means the minimum required distance between pedestrians.

The pedestrian comfort distance $L^{c}$ can be approximately equal to the reaction distance of the pedestrian demand. Therefore, the pedestrian comfort distance is a dynamic value; as the pedestrian speed increases, the required comfort distance increases; otherwise, the required comfort distance decreases:

$$
L^{c}=t_{r} V_{f} \cos \theta
$$




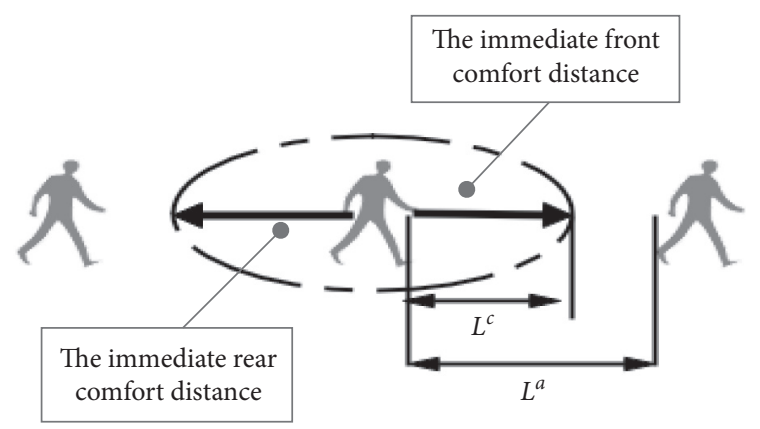

Figure 1: Comfort distance reserved among travelers.

When pedestrians interact with surrounding pedestrians to produce tangential behavior, pedestrians are usually unaware of the pedestrian situation behind them due to the limitations of human characteristics. Therefore, the principal analysis is on the interaction between pedestrians and immediate front walkers and side walkers. For followers, when the actual distance is greater than or equal to the required comfort distance, the follower may choose to increase the walking speed appropriately or maintain the same speed. When the actual distance is less than the required comfortable distance, the follower may choose to slow down or stop to avoid it. If the tangential change possibility coefficient $\delta$ is the ratio of the actual distance $L^{a}$ between pedestrians and the comfort distance $L^{c}$, then the tangential change possibility coefficient affects the number of tangential change behaviors:

$$
\delta=\frac{L^{a}}{L^{c}}
$$

When $\delta>1$, it indicates that the actual distance between the follower of the current channel and the immediate predecessor pedestrian is long, and the follower tends to keep the current channel moving. When $\delta<1$, it indicates that the actual distance between the following pedestrian in the current channel and the immediate predecessor pedestrian is short; i.e., the pedestrian walks closely to the immediate predecessor pedestrian, and the following pedestrian then tends to have a tangential change to the other channel.

The spatial changes before and after the tangent movement of pedestrians are shown in Figure 2. Before the tangent movement, the comfortable distance for pedestrian $O_{1}$ is $L_{1}^{c}(0)$ and the actual distance between pedestrian $O_{1}$ and pedestrian $\mathrm{O}_{2}$ is $L_{1-2}^{a}(0)$. After the tangent movement, at the time of $t$, the comfort distance of $O_{1}$ is $L_{1}^{c}(t)$, and the actual distance between the tangential change pedestrian $O_{1}$ and the immediate front pedestrian $\mathrm{O}_{2}$ of the target channel becomes $L_{1-2}^{a}(t)$ :

$$
L_{1-2}^{a}(t)=L_{1-2}^{a}(0)+\left[L_{O_{2}}^{h}(t)-L_{O_{2}}^{h}(0)\right]-\left[L_{O_{1}}^{h}(t)-L_{O_{1}}^{h}(0)\right] .
$$

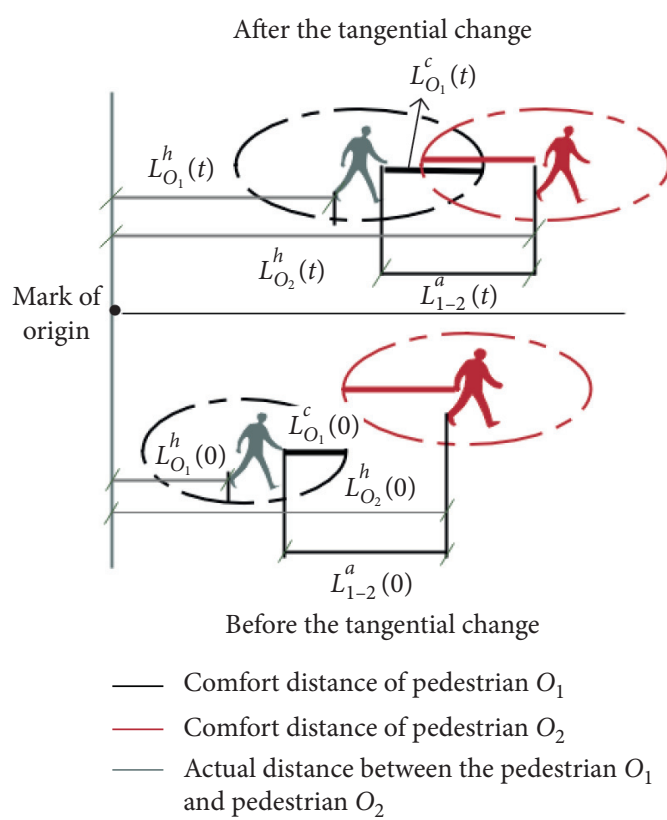

FIGURE 2: Changes in pedestrian position due to tangential change behavior interactions.

In the formula, $L_{\mathrm{O}_{1}}^{h}(t)$ and $L_{\mathrm{O}_{2}}^{h}(t)$ denote the horizontal distance of pedestrian $O_{1}$ and $O_{2}$ from the origin at moment $t, L_{O_{1}}^{c}(t)$ denotes the comfortable distance of pedestrian $O_{1}$ at moment $t$, and $L_{O_{2}}^{h}(t)$ denotes the actual distance between pedestrian $O_{1}$ and pedestrian $O_{2}$ at the moment.

2.2. Conditions for the Occurrence of a Tangential Change Behavior. Through the above analysis of the pedestrian tangential change possibility coefficient $\delta$ and the change of pedestrian position caused by the tangential change behavior, it can be concluded that when the pedestrian $O_{1}$ does not reach his desired speed and the actual distance $L^{a}$ from the pedestrian immediately in front of him in current lane $\mathrm{O}_{3}$ is less than his comfort distance, driven by his desired speed, the pedestrian $O_{1}$ tends to make a tangential movement to other lanes. However, the tangential change behavior of pedestrian $O_{1}$ mainly depends on the magnitude of the tangential change possibility coefficient $\delta$ of the target channel, i.e., whether the distance between the immediate predecessor pedestrian $\mathrm{O}_{2}$ of the target channel and the immediately following pedestrian $\mathrm{O}_{4}$ of $\mathrm{O}_{2}$ is considered to meet the minimum required distance from the tangential change of pedestrian $\mathrm{O}_{2}$ to the target channel. Further analysis in combination with (4) yields that the minimum required distance $L_{\min }$ from the tangential pedestrian change to the target channel can be derived from 


$$
L_{\min }=L_{1}^{c}(t)+\left\{\int_{0}^{\tau} \int_{0}^{\tau}\left(a_{O_{1}}(\tau)-a_{O_{2}}(\tau) \mathrm{d} \tau \mathrm{d} \tau+\left[V_{O_{1}}(0)-V_{O_{2}}(0)\right] t\right\} \cos \theta .\right.
$$

In (5), $a_{\mathrm{O}_{1}}$ and $a_{\mathrm{O}_{2}}$ are the acceleration of pedestrians $O_{1}$ and $\mathrm{O}_{2}$, respectively; $V_{\mathrm{O}_{1}}(0)$ and $V_{\mathrm{O}_{2}}(0)$ are the initial velocity before pedestrian chooses tangential change behavior of $\mathrm{O}_{1}$ and $\mathrm{O}_{2}$, respectively; and $t$ is the total time of occurring tangential change behavior.

Thus, the tangential change behavior of pedestrian $O_{1}$ is related to the pedestrians $O_{1}$ and $O_{2}$ 's acceleration and the initial velocity before lane change, as well as the time spent on lane change and comfort distance of pedestrian $O_{1}$. The conditions for tangential pedestrian change vary dynamically depending on the difference of these factors. If pedestrian $O_{1}$ wants to change lane to the target channel, this condition must be satisfied:

$$
L^{c}>L_{\min }
$$

When the tangential change possibility coefficient $\delta$ is large, it indicates that there are few pedestrians in the area, and also the condition is met for pedestrian change lane. However, in fact, few pedestrians choose tangential change behavior in this case because the pedestrian's movement is unrestricted and the pedestrian can walk at or near the desired speed. When the tangential change possibility coefficient $\delta$ is close to 1 , there are more pedestrians in the area, and the pedestrian walking space is compressed, so pedestrians cannot freely pass and frequently slow down; instead, they often tangentially change to other lane. Tolerance $\lambda$ is thus introduced to describe the tolerance time that a pedestrian can accept when forced to slow down when the channel is congested. That is, the pedestrian's actual speed is less than the desired speed and is always decelerating within their tolerance $\lambda$. The pedestrian will choose to tangentially change to another channel. The pedestrian deceleration state is recorded in time steps, and the pedestrian tolerance decreases by 1 for each deceleration, so the control condition for pedestrian changes lane is

$$
\lambda<0 \text {. }
$$

\section{Multichannel Crowd Evacuation Model and Simulation}

3.1. Pedestrian Simulation Model. Blue and Adler [17] believed that the basic movement of pedestrians was mainly forward movement and tangential change conflict handling. Therefore, the tangential behavioral passage model focused on forwarding motion rules and conflict-handling rules, and they made the following assumptions.

Assumption 1. Without the effect of tangential change behavior, pedestrian $O_{j}$ walks at the same speed $V_{j}^{a}$ in the same lane, and pedestrian $O_{j}$ usually walks at his desired speed $V_{j}^{e}$.
Assumption 2. After pedestrian $O_{1}$ enters the target track $T_{g}$, the walking speed $V_{\mathrm{O}_{2}}$ remains consistent with the speed $V_{\mathrm{O}_{2}}$ of the target track $T_{g}$, and the tangential change behavior does not affect the speed of the immediate predecessor pedestrian in the target track $T_{g}$ but has some influence on the speed of the immediate successor pedestrian.

Assumption 3. The speed at which the pedestrian moves depends not only on the maximum forward demand speed but also on the free distance in the direction to move forward [17]. When the pedestrian's direction of advance is obstructed, the pedestrian has a certain tolerance $\lambda$ to the surroundings, and the pedestrian's tolerance $\lambda$ is acceptable to the pedestrian of the maximum forced deceleration tolerance time and is consistent with the maximum acceptable wait time for pedestrians to cross the street.

Assumption 4. Pedestrian $O_{1}$ tends to select tangential change and maintain a constant acceleration as he or she changes from the current channel to the target channel $T_{g}$. After a successful tangential change to lane, pedestrian $O_{1}$ gradually keeps in line with the velocity $V_{\mathrm{O}_{2}}$ of the immediate predecessor pedestrian $\mathrm{O}_{2}$.

Pedestrians have the habit of walking at their desired speed, and the speed of the pedestrians on the target channel is constant; i.e., $a_{\mathrm{O}_{2}}=0$ and $V_{\mathrm{O}_{2}}(0)=V_{\mathrm{O}_{2}}(0)$. When the pedestrian enters the target channel, he or she will adjust his or her speed to match the speed of the immediately preceding pedestrian; i.e., $V_{\mathrm{O}_{2}}(t)=V_{\mathrm{O}_{2}}(t)$. In the process of tangential change, pedestrian $O_{1}$ opts for uniform acceleration and then $V_{O_{1}}(t)=V_{O_{1}}(0)+a_{O_{1}}$. After simplifying (5) and combining it with (2), we get the minimum demand distance of the pedestrian tangential change to the target channel:

$$
L_{\min }=\left\{t_{r} V_{O_{1}}(t)-\frac{1}{2}\left[V_{O_{2}}(0)-V_{O_{1}}(0) t\right]\right\} \cos \theta .
$$

When the tolerance $\lambda$ is less than 0 and the conditions of the permissible tangential change are met, pedestrians begin to seek and make a tangential change to the target lane. Lu et al. [18] adopted a survival analysis to derive the maximum waiting time for pedestrians to cross the street as 40-50 s. Based on the measured data of signalized intersections in Beijing, Guo et al. [19] derived the acceptable waiting time for pedestrians as $50 \mathrm{~s}$. From hypothesis 3, the tolerance level could be obtained as $\lambda_{j}=50(s)$.

The walking speed of pedestrian $j$ at time step is denoted by $V_{j}^{a}(t)$. The average evacuation speed for the number of people $(N)$ in line could be expressed as

$$
\overline{V_{N}}=\frac{1}{N} \sum_{j=1}^{N} \sum_{t=0}^{T} V_{j}^{a}(t), \quad \mathrm{d}(t=0,1,2, \ldots, T) .
$$


When the evacuation distance is $S$ and the number of rows is $N$, the evacuation time is

$$
T_{N}^{t}=\frac{S}{\overline{V_{N}}}
$$

3.2. Simulation Rules. According to the above-mentioned passage model, the simulation rules of pedestrian tangential change behavior are established by using the cellular automaton method, taking into account the movement characteristics of the passage pedestrians.

Rule 1. If $\theta \neq 0$, the process of tangential-normal motion in a three-dimensional nonhorizontal channel is reduced to tangential motion in a two-dimensional plane, and the tangential-normal velocity of the pedestrian has a "projection" as tangential velocity [11].

Rule 2. The rectangular area of the two-dimensional channel plane is discretized into a cellular space with edge length $d$. The pedestrian cell is set to move from left to right to simulate the pedestrian interaction process in the longdistance channel. As shown in Figure 3, the blank cell is a self-organizing pedestrian formation of the channel. The red full line cell is a wall or handrail railing, which is off-limits to entry, while the red dotted line cell represents the gap between two pedestrian channels, which may be passed through or occupied.

Rule 3. Before the pedestrian selects the tangential change behavior, he or she should first select the adjacent channel as the target channel. The pedestrian can only have a tangential change to the target channel if the tangential change probability coefficient of the target channel is $\delta>1$. As shown in Figure 3, when the pedestrian $O_{1}$ has left and right two adjacent channels, one of them can be selected at random, each with the probability of selection for 0.5 . When pedestrians $\mathrm{O}_{2}$ and $\mathrm{O}_{3}$ both choose the middle stock channel as the target stock channel, the probability of success for each is 0.5 . After one of the people makes a tangential change to the target channel, the other person slows down or stops. After the pedestrian changes to lane, the tolerance $\lambda$ is set as its maximum value.

Rule 4. The states of the cell are vacant or occupied, and each cell can only be occupied by one pedestrian. Pedestrians on the middle channel have three directions of advancement: lower, lower left, and lower right. Pedestrians on both sides of the channel have two directions of advancement: lower, lower left (or lower right), as shown in Figure 4:

Rule 5. The initialization phase sets up $N$ pedestrians randomly distributed on the channel, with pedestrians having different expected velocities, but the same initial velocity, acceleration, and deceleration. Pedestrians move forward at their speed within each time step. Before moving, they will determine if the cellular ahead is occupied by other pedestrians. If it is congested, pedestrians can choose to slow

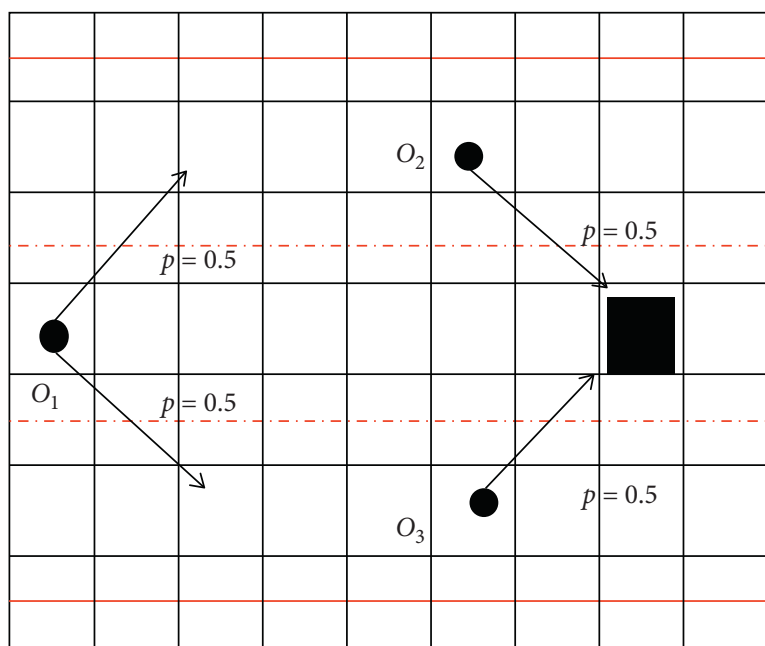

The direction of motion

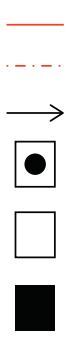

A wall or handrail railing

. . The gap between two pedestrian channels

$\longrightarrow$ The direction of the tangential change

The pedestrian cellular cell

The blank cellular cell

The conflicting cellular cell

FIgURE 3: A two-dimensional cellular space for pedestrian tangential change behavior.
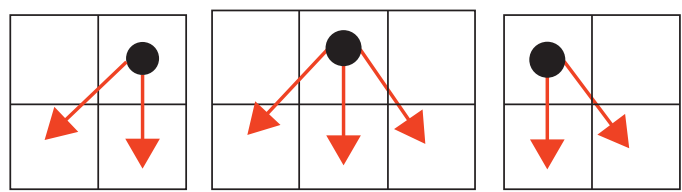

Figure 4: Pedestrian direction of travel.

down or stop. For each deceleration or stop, the tolerance decreases by 1 . When the pedestrian's tolerance $\lambda<0$, the pedestrian chooses the tangential change behavior. If it is clear, pedestrians can accelerate in the original lane.

3.3. Case Studies. Take the evacuation drill in the J3 teaching building of China Three Gorges University as an example to establish the access scenario and the pedestrian subject, and simulate the interaction process when the pedestrian moves according to the five simulation rules mentioned above (see Figure 5). Disperse the channel region into $F \times W$ cells, with $F=41$ and $W=7$; set the cellular edge length as $d=0.3 \mathrm{~m}$; simulate the channel length as $12.3 \mathrm{~m}$, width as $1.8 \mathrm{~m}$, and maximum channel capacity as $N=123$ people; then, three people can be accommodated side by side (number of channels $m=3$ ).

Walking speed correlation studies have shown that the maximum walking speed for pedestrians is $1.5 \mathrm{~m} / \mathrm{s}$ [20], the normal walking speed for pedestrians in subway 


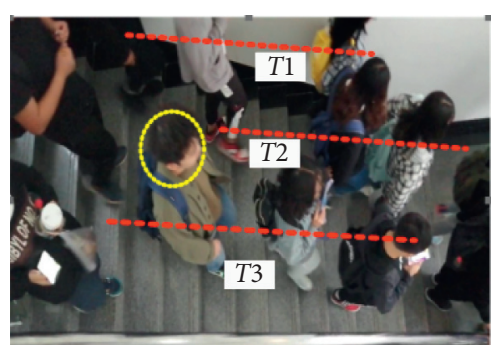

(a)

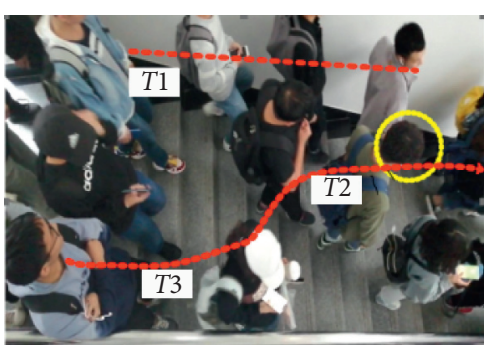

(b)

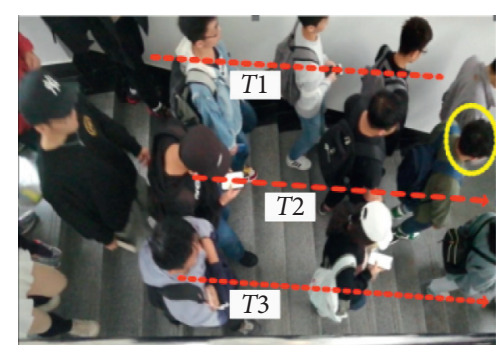

(c)

Figure 5: Interactive process of tangential pedestrian behavior when descending stairs: (a) following process; (b) tangential process; (c) following process.

interchanges is $0.8 \mathrm{~m} / \mathrm{s}$ [21], and the average walking speed for mixed sexes is $1.2 \mathrm{~m} / \mathrm{s}$ [22]. By correcting for the expected speed of the crowd, the evacuation speed was calculated to be $1.43 \mathrm{~m} / \mathrm{s}$ for males and $1.37 \mathrm{~m} / \mathrm{s}$ [23] for females. Besides, the speed of pedestrian movement decreases as the slope increases [24]. Therefore, set the maximum desired pedestrian speed between $1.2 \mathrm{~m} / \mathrm{s}$ and $1.5 \mathrm{~m} / \mathrm{s}$, with an initial speed of $1.2 \mathrm{~m} / \mathrm{s}$ for the horizontal corridor $s$, while the initial velocity of the nonhorizontal channel is $0.7 \mathrm{~m} / \mathrm{s}$.

Pedestrian density correlation studies have shown [25] the following: (1) Low density is the density below one person $/ \mathrm{m}^{2}$. (2) Medium density state the density around two people $/ \mathrm{m}^{2}$. (3) High-density state is the density above 3 people $/ \mathrm{m}^{2}$. In the event of an emergency, evacuation of crowds will flood the corridor and pedestrian flow will change from a low-density to a high-density state. Therefore, the stimulated pedestrian density is 1 person $/ \mathrm{m}^{2}, 2$ people $/ \mathrm{m}^{2}$, 3 people $/ \mathrm{m}^{2}$, and 4.7 people $/ \mathrm{m}^{2}$, and the corresponding pedestrian number is 22 people, 44 people, 66 people, and 105 people. Set the angle and the horizontal and nonhorizontal channels, respectively, to simulate the evacuation at different stages of the evacuation process.

Set the initial number of pedestrians $N$ randomly distributed in the channel area to form three channels with a minimum pedestrian demand distance of $L^{a}=t_{r} V_{f} \cos \theta+l_{\min }$. The behavioral options include the following: (1) When the actual distance to the pedestrian is less than the comfortable distance, the pedestrian chooses to slow down or stop. (2) Furthermore, if the pedestrian tolerance $\lambda<0$ and the target channel satisfies the tangential change condition, then the tangential change behavior is selected. The specific pedestrian movement rules are shown in Figure 6, the simulation uses time steps (ticks) as the unit of timing, and a time step equals 0.25 second, which corresponds to a pedestrian tolerance of 200 (ticks).

\section{Simulation Results and Analysis}

Set the simulation time to 300 seconds, which is equivalent to $5 \mathrm{~min}$. The trajectory of the pedestrian is recorded during the pedestrian interaction, and after 300 seconds evolution, the distribution and tangential change of pedestrians in the channel region are shown in Figure 7.

From the pedestrian movement trajectories recorded in Figure 7, it can be seen that when the pedestrian density is high and the tangential change condition is met, the tangential change behavior is quite frequent. With the gradual increase from low density to high density, the interaction behavior is characterized by the evolution of "no tangential change $\longrightarrow$ occasional toggling $\longrightarrow$ frequent toggling $\longrightarrow$ following closely." The tangential change pedestrians are generally less attentive to the status of predecessorfollowing pedestrians in the target channel and are prone to position conflicts in high densities. When a tangential change pedestrian is obstructed by following pedestrian of the target channel, he will choose to walk briefly in the gap and then wait for the opportunity of tangential change to the target channel. When the following pedestrian of the target channel does not affect the tangential change pedestrian, the latter will quickly tangentially change to the target channel, and the former will slow down to avoid a collision. Based on the above behavioral pattern analysis, it can be seen that irrational tangential change of multichannel road evacuation pedestrians is very likely to cause position conflicts with predecessor pedestrians, leading to interpersonal collisions, falls, and even trampling accidents. In particular, when the distance between the left and right pedestrians is narrow, with the location conflicts caused by the tangential change behavior lack of appropriate buffer space, resulting in congestion, the predecessor pedestrian "inertia" speed is very likely to cause trampling accidents. In view of this, it is recommended that when organizing an evacuation, it is preferable to choose a track against a wall and handrail, which will provide a sense of psychological safety for pedestrians and enough space for pedestrians in the middle stockade to deal with irrational tangential change behavior.

We use the shear frequency, that is, the total times of pedestrian entering or exiting each channel, to analyze the pedestrian's tangential behavior over 300 seconds.

Figure 8 shows the upward and downward trend in the number of pedestrians with pedestrians entering or exiting channel. When the number of people in one channel decreases and the number of people in the other channels does not increase, the pedestrian is adjusting his location and is currently located between two channels. By combining Figures 7 and 8, it is clear that the exogenous factor influencing pedestrian tangential change behavior is pedestrian flow density. Pedestrians usually enter from a larger number of channels to a smaller number of channels. The pedestrians in Figure 8(a) rarely engage in tangential change 


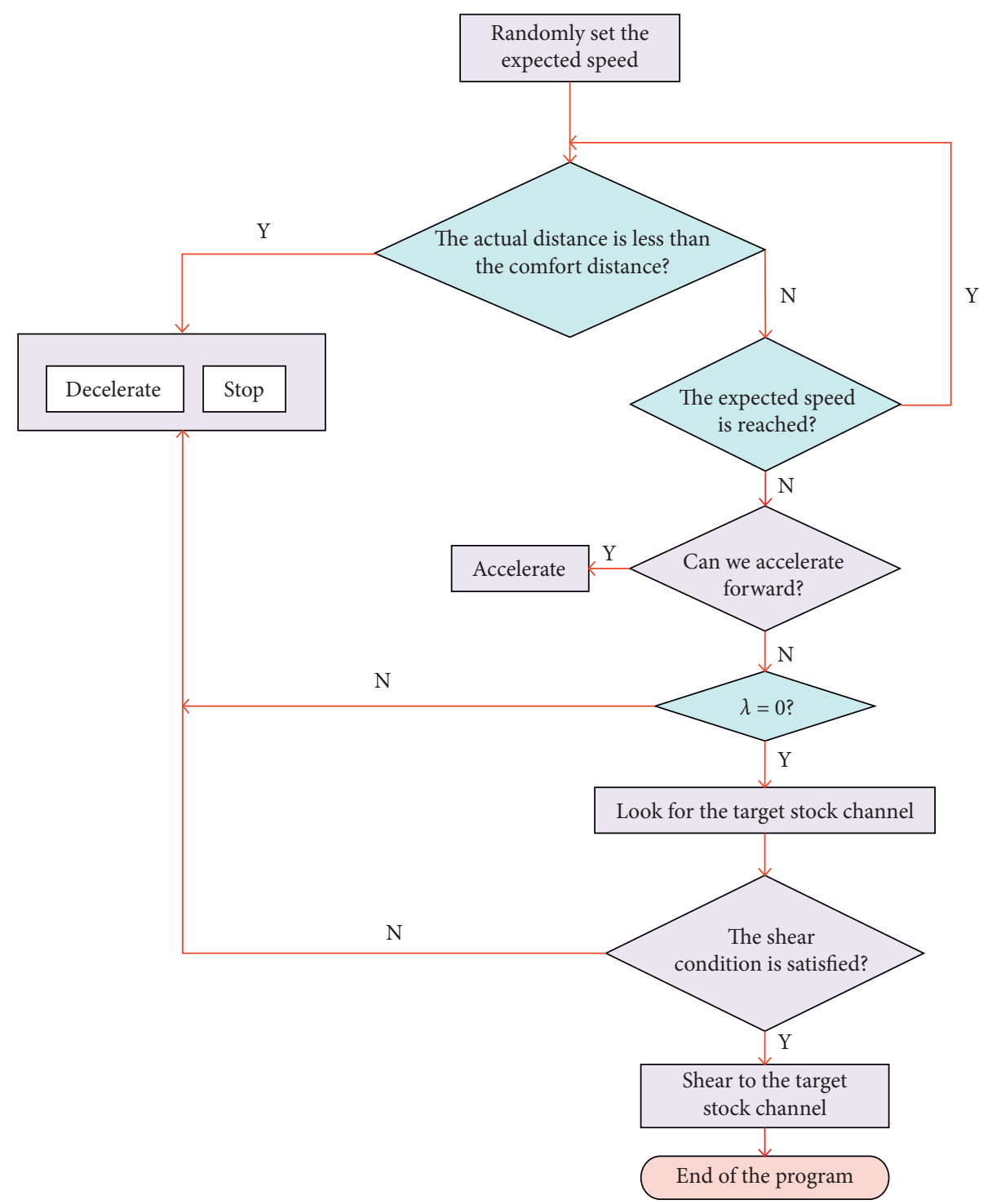

FIGURE 6: Pedestrian movement rules flowchart.

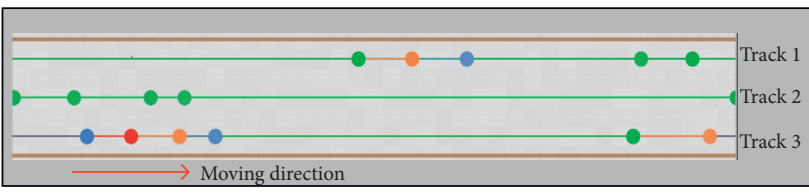

(a)

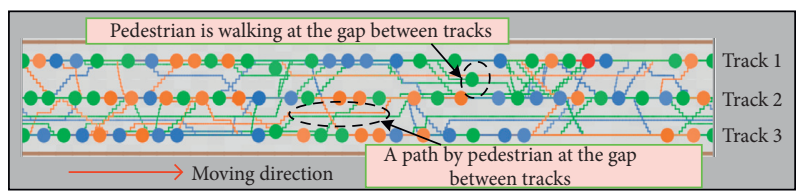

(c)

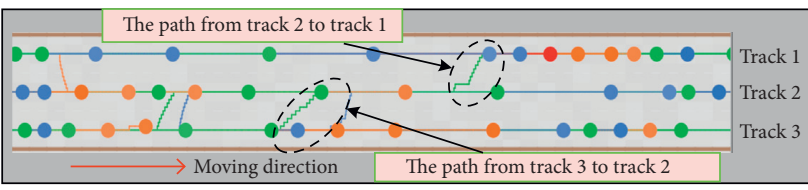

(b)

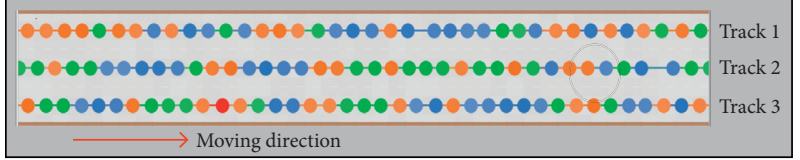

(d)

Figure 7: Pedestrian distribution and movement trajectory statistics. For the corridor area after 300 seconds: (a) $N=15$, (b) $N=44$, (c) $N=85$, and (d) $N=115$.

behavior, mainly because when the density is one person $/ \mathrm{m}^{2}$ $(N=22)$, the pedestrian space is quite large, local pedestrian velocities are similar, and pedestrian movement is unrestricted, allowing the pedestrians to freely accelerate, decelerate, and maintain a higher walking speed. As pedestrian flow density increases, pedestrian speed is impeded, 

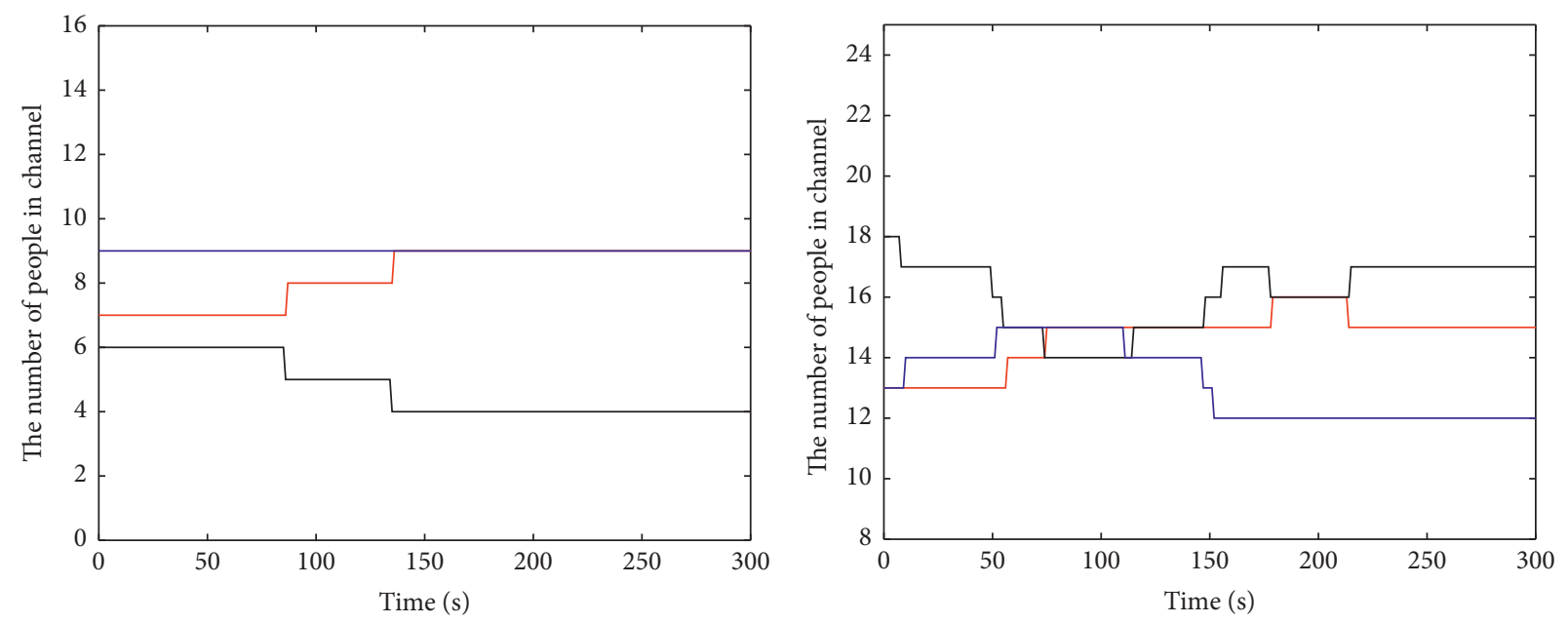

- Track 1
Track 2
- Track 3

Track 1

- Track 2

- Track 3

(a)

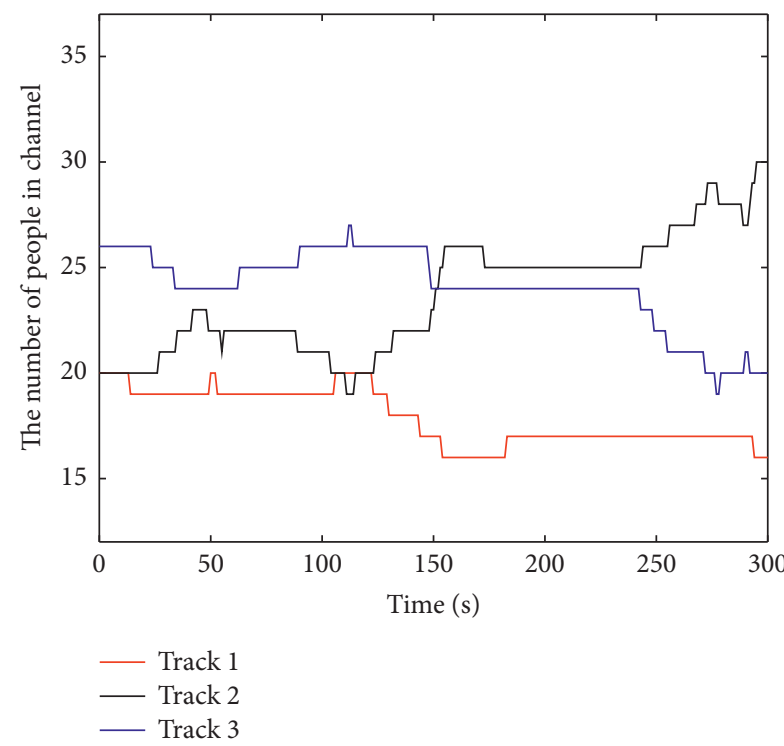

(c)

(d)

Figure 8: Change in the number of people $N$ in each channel over time: (a) $\rho=1.0$; (b) $\rho=2.0$; (c) $\rho=3.0$; (d) $\rho=4.7$.

and pedestrian tolerance $\lambda$ continues to decrease, so pedestrians attempt to increase walking speed by changing their track to other tracks, resulting in tangential change behavior of pedestrian frequently cropping up, as shown in Figures $8(\mathrm{~b})$ and $8(\mathrm{c})$. In crowded conditions, however, pedestrians can only choose to follow the immediate predecessor closely, resulting in a tangential change probability factor $\delta$ close to 1 and a significant reduction in tangential change behavior, as shown in Figure $8(\mathrm{~d})$.

Figures 8 and 9 are combined to analyze the influence of tangential change behavior on the velocity of the passage. At a micro level, within 50 seconds and 100 seconds in Figure $8(\mathrm{a})$, the pedestrians in channel 2 enter channel 1 , with no significant increase in the speed of channel 2 , but a significant decrease in the speed of channel 1. Around 50 seconds in Figure 8(b), the pedestrians in channel 2 enter channel 1 and channel 3, with little change in the velocity of channel 2, but an immediate decrease in the velocity of channel 1 and channel 3. It can be seen that, for the lane pedestrian exit, the tangential change behavior favors the growth rate of the immediate predecessor pedestrian and the successor pedestrian. For the lane pedestrian enter, it is easy to cause the following pedestrians to suddenly slow down or even stop avoiding. The higher the following pedestrian speed is, the greater the susceptibility of pedestrian to tangential change behavior will be. In the macroscopic view, the tangential change behavior causes the pedestrian speed to be unstable, in Figures 9(b) and 9(c), the channel velocity fluctuates considerably, and the velocity of each channel also differs greatly. As pedestrian density increases and 


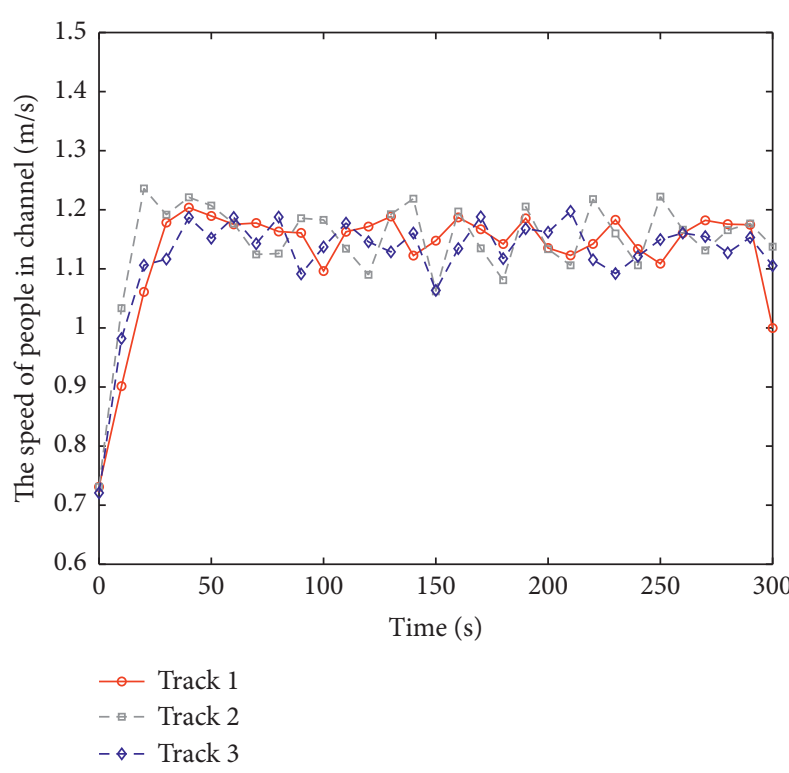

(a)

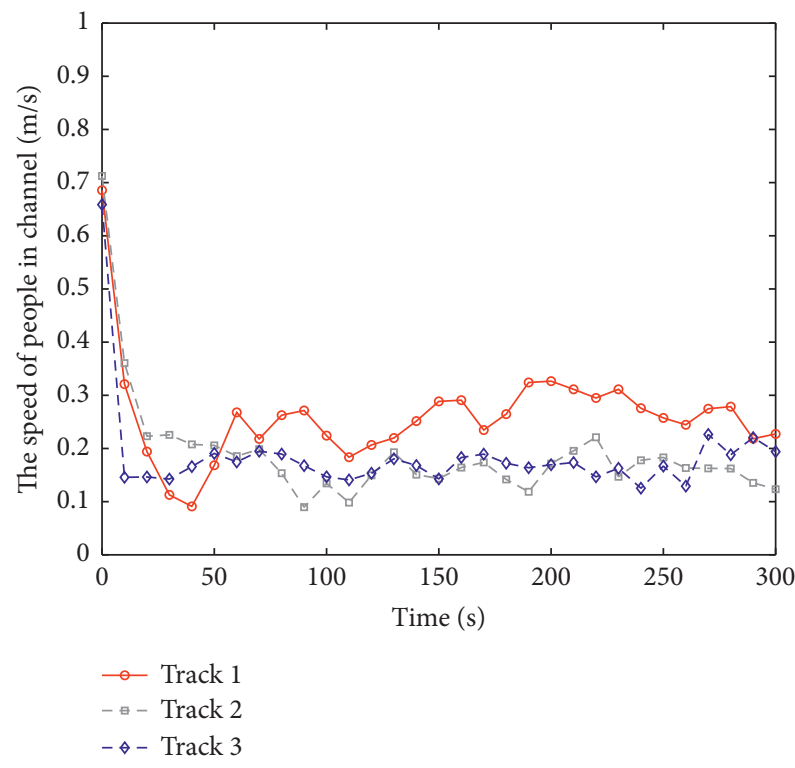

(c)

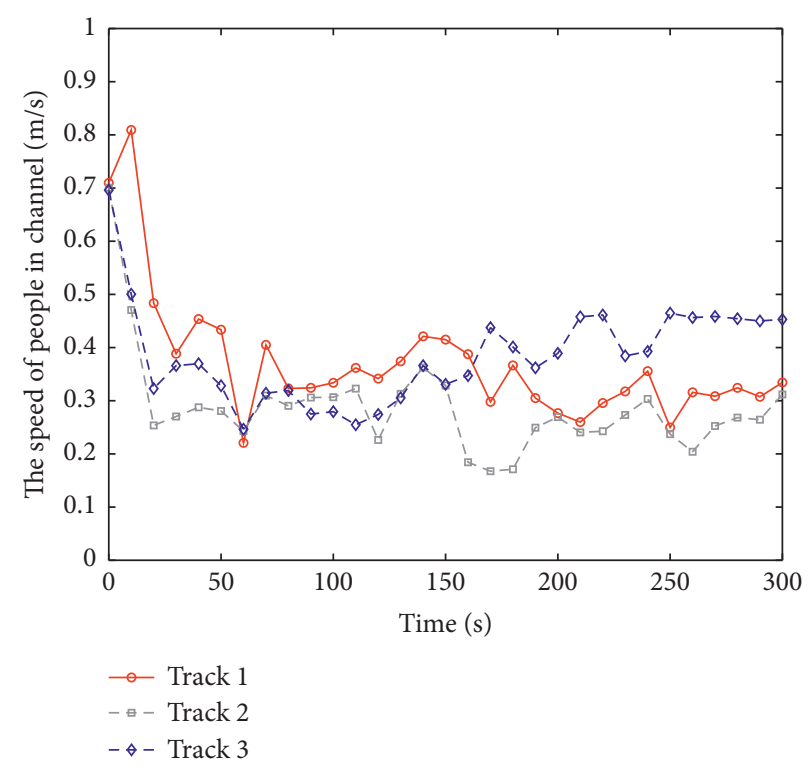

(b)

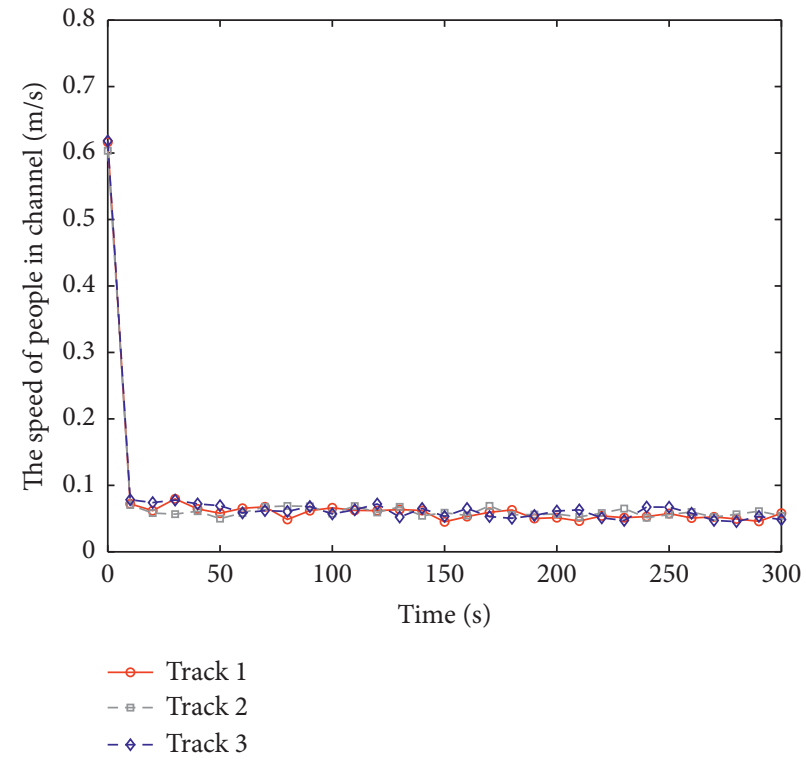

(d)

Figure 9: Changes in pedestrian velocity over time by channel: (a) $\rho=1.0$; (b) $\rho=2.0$; (c) $\rho=3.0$; (d) $\rho=4.7$.

pedestrian tangential change behavior utility decreases, the speeds of the channels gradually remain the same, Figure 9(d).

In order to study the difference in walking speed when pedestrian is allowed to make the shear behavior and is restricted in the horizontal channel $(\theta=0)$ and the nonhorizontal channel $\left(\theta=26^{\circ}\right)$, the relationship between the average walking speed and density of pedestrians is obtained by simulating the process of pedestrian with different numbers of pedestrians for each case; simulation duration is $300 \mathrm{~s}$. It reveals the mechanism of the effect of tangential change behavior on the velocity and density of the multichannel crowd, as shown in Figure 10.
As shown in Figure 10(a), the threshold at which tangential change behavior affects the speed of the horizontal channel crowd is $\rho=1.3$. At $\rho<1.3$, the passage velocity after a tangential change is higher than the passage velocity when following. At $\rho>1.3$, the passage speed of following is instead slightly higher than the passage speed after the tangential change. When pedestrian density reached 4.5 persons $/ \mathrm{m}^{2}$, there was no significant difference in the benefits of the two strategies for pedestrians choosing to change or follow. In Figure 10(b), the threshold for the effect of tangential change behavior on the velocity of the nonhorizontal channel crowd is $\rho=1.5$. Compared to the horizontal channel, the passage speed after a tangential change is also higher than the 


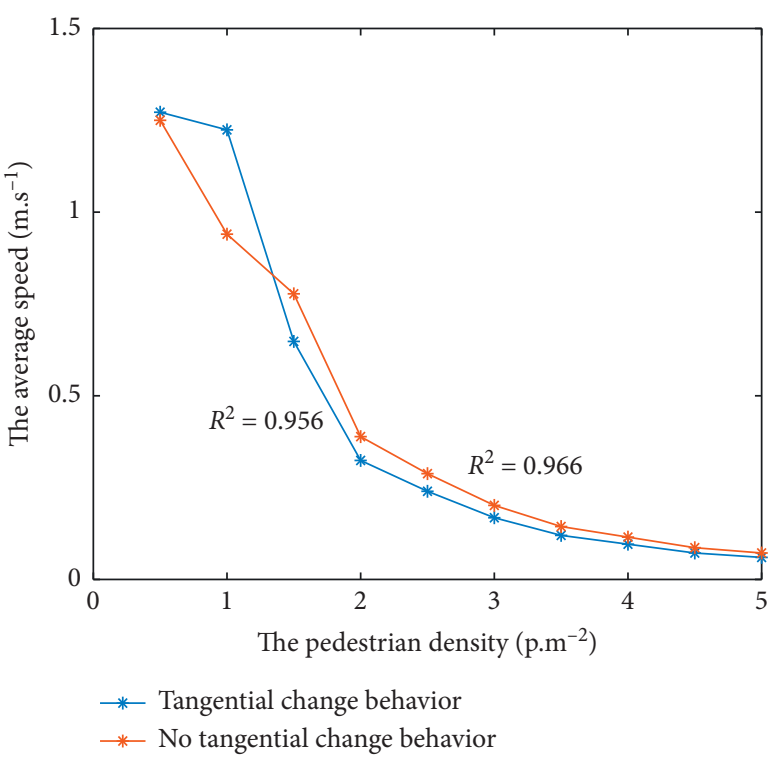

(a)

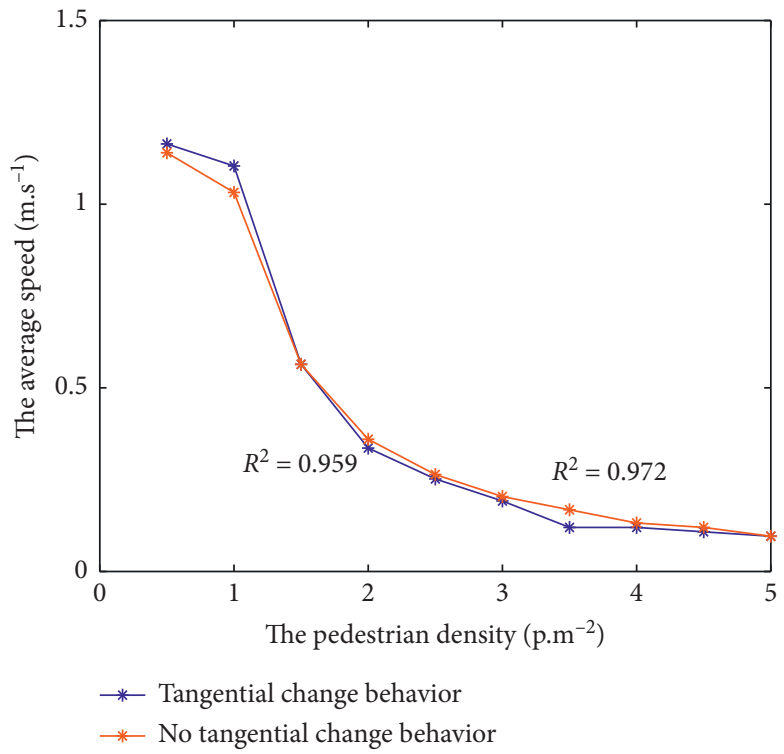

(b)

Figure 10: Curves of the effect of tangential change behavior on the speed and density of a multichannel crowd: (a) $\theta=0$; (b) $\theta=26^{\circ}$.

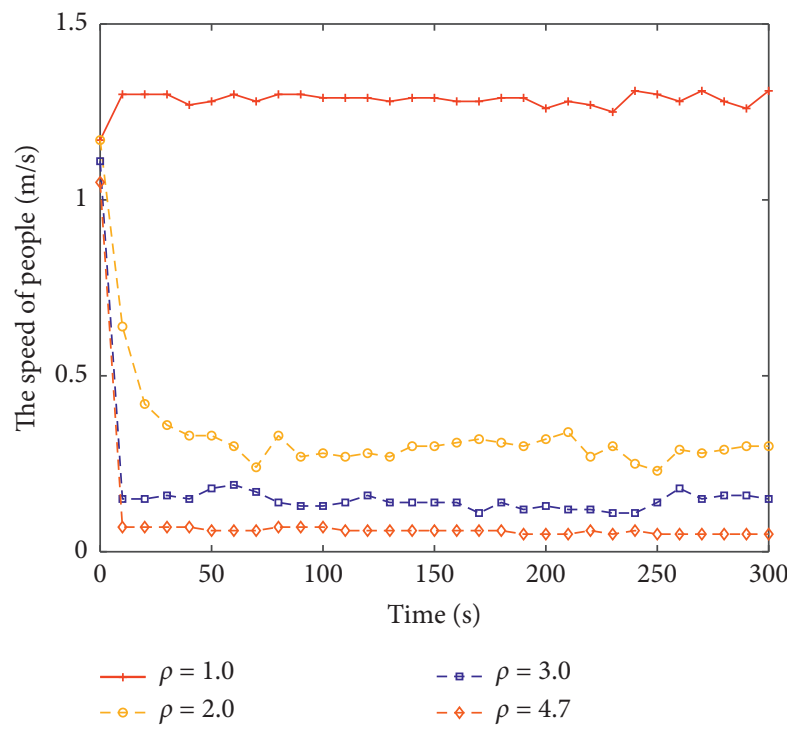

(a)

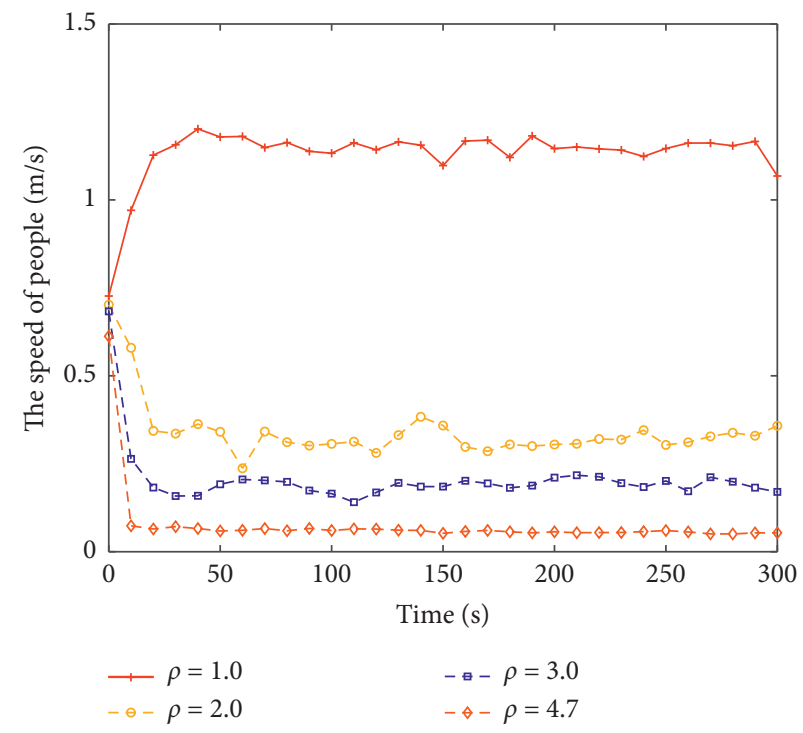

(b)

FIGURE 11: Variation in average pedestrian speed at different densities: (a) the horizontal channel $\theta=0$; (b) the nonhorizontal channel $\theta=26^{\circ}$.

passage speed of following at density $\rho<1.5$. However, when the density $\rho>1.5$, unlike the velocity changes in the horizontal channel, the two strategies in the nonhorizontal channel both have very approximate walking speed. The above results suggest that the strategy of following the person in front is better than the choice of overtaking when the pedestrian density is greater than a threshold in general. This conclusion is the opposite of what pedestrians are trying to do when increasing speed by tangential change behavior, proving that "haste brings no success."
Comparing Figures 11(a) and 11(b), it can be concluded that the shear behavior causes the average speed of pedestrians to fluctuate, and the speed in horizontal and nonhorizontal corridors varies in the same pattern. When $\rho=1.0$, the average speed of all pedestrians increased significantly, but the horizontal channel average speed was higher than the nonhorizontal channel average speed, and the horizontal angular effect was significant. When $2.0 \leq \rho \leq 4.7$, the average speed of all pedestrians decreased visibly, and the horizontal angle $\theta$ had no obvious effect on speed. When $\rho=4.7$, the velocity of the nonhorizontal 


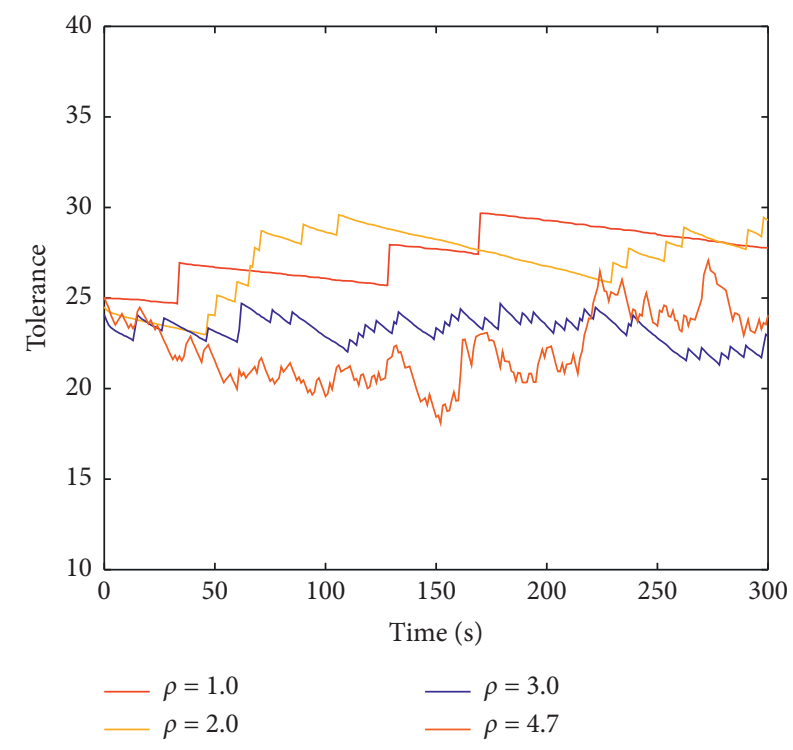

(a)

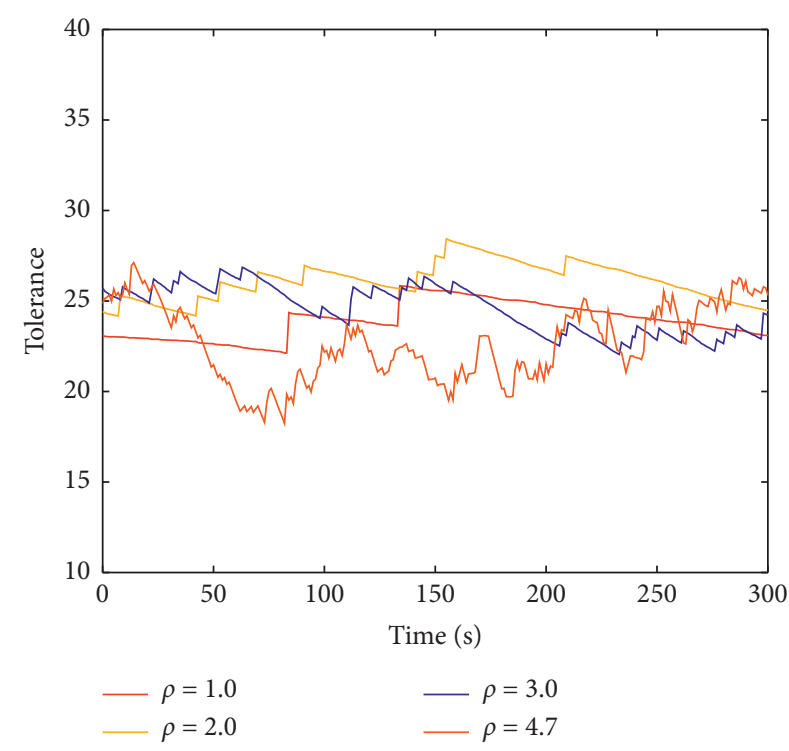

(b)

FIgURE 12: Changes in pedestrian tolerance at different densities: (a) the horizontal channel $\theta=0$; (b) the nonhorizontal channel $\theta=26^{\circ}$.

channel is slightly higher than that of the horizontal channel. As the horizontal angle $\theta$ affects the comfortable distance for pedestrians, the distance between pedestrians in horizontal corridors is larger, so pedestrians can easily slow down compared to the situation in nonhorizontal corridors. As a result, the frequency of tangential change behavior should be greater for the horizontal channel than for the nonhorizontal channel.

According to Figure 12, tolerance $\lambda$ is related to the tolerance time for pedestrians to slow down and the tangential change behavior. That is, the longer the pedestrian slows down, the less tolerance the pedestrian has. The pedestrian tolerance will be "full-blooded" reaching 50 seconds after the tangential change behavior, and the average pedestrian tolerance will increase significantly. In some cases, the tolerance curve rises or falls in a stepwise slope, indicating that at first pedestrian deceleration leads to a slight decrease of tolerance, and then the pedestrian selects a new target channel resulting in a sudden tolerance increase. The smaller the reduction of tolerance, the less restricted the pedestrian. The results show that at $\rho=4.7$ pedestrian tolerance is the lowest and varies frequently but shows an overall downward and then upward trend. Accordingly, the average speed of the pedestrians is close to 0 , indicating that the pedestrians were in a very crowded situation. Limited by reality, at this point, pedestrians can only move intermittently, but they are still looking for target channels and eager to increase speed. Horizontal corridor pedestrians have more variation of tolerance due to frequent tangential change behavior compared to nonhorizontal corridor pedestrians.

\section{Conclusion}

In this paper, a multichannel crowd evacuation model is constructed by coupling the two influencing factors of dynamic comfort distance and tolerance. It organizes the rules of forwarding pedestrian movement and conflict-handling rules and uses the cellular automaton for simulation analysis to investigate the multistock way pedestrian behavior. The mechanism of the effect of tangential change behavior and its interaction on evacuation speed provides theoretical guidance for the evacuation organization of multichanneled crowds. The research findings are as follows:

(1) The Evolution of Tangential Change Behavior and Its Interactions. The tangential change behavior of the pedestrian and its interaction occur when the immediate predecessor pedestrian is slow, and the pedestrian choice to change lane results in passive deceleration of the predecessor pedestrian in the target channel. By simulating the trajectory of pedestrians, it is found that tangential pedestrian behavior shows the evolution of "no tangential change $\longrightarrow$ occasional tangential change$\longrightarrow$ frequent tangential change $\longrightarrow$ following closely." Pedestrian tangential change is more frequent when the pedestrian walking space is compressed, and the direction of forward motion is obstructed. When pedestrian making tangential change behavior has a positional conflict with a predecessor pedestrian in the target channel, it relies heavily on the respective instantaneous reactions of the tangential change pedestrian and the predecessor pedestrian, who will choose to walk at the channel gap. Therefore, an evacuation that ensures the required space for pedestrians to walk at the tracks gap, while not affecting the normal flow of pedestrians on both sides, can reduce the occurrence of trampling accidents.

(2) Achieving Modulation of the Velocity-Density Relationship by Tangential Change Behavior. When $\rho<1.3$, the tangential change behavior of pedestrians 
in horizontal corridors can significantly increase speeds compared to nonhorizontal corridors. The horizontal angle $\theta$ mainly affects the comfortable distance for pedestrians and widens the comfortable distance for pedestrians in horizontal corridors. At the same density, horizontal channel pedestrians are more likely to choose tangential change behavior than nonhorizontal channel pedestrians. In addition, this paper proposes pedestrian tolerance as an internal driver of pedestrians' choice of whether to tangentially change their location or not, which can better reveal the interactive mechanism of pedestrians' tangential change behavior when they are in different environments.

(3) Follow-Up Study. In the case of unorganized evacuation, there may be both opposite and converging pedestrian flows, making the tangential behavior and its interaction mechanism influenced by pedestrian hedging, behavioral convergence, rule compliance, exit distance, etc., which need to be further studied.

\section{Data Availability}

The data used to support the findings of this study are included within the article.

\section{Conflicts of Interest}

The authors have no conflicts of interest regarding the publication of this paper.

\section{Acknowledgments}

This study was supported by the 2019 Open Fund of Hubei Key Laboratory of Hydropower Engineering Construction and Management (China Three Gorges University) (2019KSD05) and the National Natural Science Foundation of China (51878385).

\section{References}

[1] L Zhao and J. Ma, "Risk analysis of stampede by dynamic Bayesian network," China Safety Science Journal, vol. 27, no. 7, pp. 157-162, 2017.

[2] H. Yue, X. Zhang, G. Chen et al., "Simulation study of evacuated pedestrian flow with unbalanced initial location layout," Journal of Physical Science, vol. 61, Article ID 130509, 2012.

[3] V. J. Kok, M. K. Lim, and C. S. Chan, "Crowd behavior analysis: a review where physics meets biology," Neurocomputing, vol. 177, pp. 342-362, 2016.

[4] Y. Han and H. Liu, "An application of a path selection model based on a collection of evacuation paths for crowd evacuation simulation," Chinese Journal of Computers, vol. 41, pp. 2653-2669, 2018.

[5] B. Hua, Q. Liang, S. Liu et al., "Crowd acceleration anomaly detection system for public places," Journal of Safety and Environment, vol. 17, pp. 1043-1048, 2017.

[6] F. T. Illiyas, S. K. Mani, A. P. Pradeepkumar, and K. Mohan, "Human stampedes during religious festivals: a comparative review of mass gathering emergencies in India," International Journal of Disaster Risk Reduction, vol. 5, pp. 10-18, 2013.

[7] L. Huang, T. Chen, Y. Wang, and H. Yuan, "Congestion detection of pedestrians using the velocity entropy: a case study of Love Parade 2010 disaster," Physica A: Statistical Mechanics and Its Applications, vol. 440, pp. 200-209, 2015.

[8] M. Moussaid, E. G. Guillot, M. Moreau et al., "Traffic instabilities in self-organized pedestrian crowds," PLoS Computational Biology, vol. 8, no. 3, 2012.

[9] J. K. K. Yuen and E. W. M. Lee, "The effect of overtaking behavior on unidirectional pedestrian flow," Safety Science, vol. 50, no. 8, pp. 1704-1714, 2012.

[10] S. Heliövaara, T. Korhonen, S. Hostikka, and H. Ehtama, "Counterflow model for agent-based simulation of crowd dynamics," Building and Environment, vol. 48, pp. 89-100, 2012.

[11] C. Yang, Q. Chen, and L. Chen, "Modeling and simulation of pedestrian flows considering the characteristics of pedestrian following behavior under visibility constraints," Journal of Physical Science, vol. 68, Article ID 240504, 2019.

[12] H. Chen, L. Zhang, P. Yang et al., "Studies on pedestrian evacuation model and simulation for limited visibility," Complex Systems and Complexity Science, vol. 3, pp. 19-25, 2014.

[13] X. Weng, D. Huang, and S. Li, "Pedestrian traffic simulation performance analysis for public buildings," Journal of South China University of Technology (Natural Sciences Edition), vol. 40, pp. 45-49, 2012.

[14] H. Yue, C. Shao, X. Chen et al., "Simulation study of opposing pedestrian traffic flow based on cellular automata," Journal of Physical Science, vol. 57, pp. 6901-6908, 2008.

[15] M. Bocian, J. H. G. Macdonald, J. F. Burn, and D. Redmill, "Experimental identification of the behaviour of and lateral forces from freely-walking pedestrians on laterally oscillating structures in a virtual reality environment," Engineering Structures, vol. 105, pp. 62-76, 2015.

[16] H. Wu, Z. Yuan, H. Li et al., "Simulation model of metro station stairway accessibility considering pedestrian heterogeneity," Transportation Systems Engineering and Information, vol. 16, pp. 141-147, 2016.

[17] V. Blue and J. Adler, "Bi-directional emergent fundamental pedestrian flows from cellular automata microsimulation," Transportation and Traffic Theory, vol. 14, pp. 235-254, 1999.

[18] S. Lu, H. Wang, and X. Liu, "A study of maximum waiting time for pedestrian crossings Based on survival analysis," Traffic Information and Safety, vol. 27, pp. 69-71, 2009.

[19] H. Guo, Z. Gao, and X. Zhao, "Pedestrian crossing behavior based on continuous models," Journal of Jilin University (Engineering Edition), vol. 39, pp. 35-40, 2009.

[20] K. Wang, M. Liao, X. Meng et al., "Traffic characteristics of stairs in shanghai metro station," Journal of Shanghai Maritime University, vol. 30, pp. 69-73, 2009.

[21] W. Yan, J. Wang, H. Xu et al., "Dynamics analysis of nanjing metro interchange stairway flow system based on measured data," Journal of Safety and Environment, vol. 17, no. 2, pp. 630-635, 2017.

[22] J. N. Laplante and T. P. Kaeser, "The continuing evolution of pedestrian walking speed assumptions," Institute of Transportation Engineers, ITE Journal, vol. 74, p. 32, 2004.

[23] X. Zheng, L. Xiang, L. Jin et al., "Subway station evacuation speed correction model and simulation considering humancyclic factors," Chinese Journal of Security Sciences, vol. 27, pp. 19-24, 2017. 
[24] T. Fujiyama and N. Tyler, "Predicting the walking speed of pedestrians on stairs," Transportation Planning and Technology, vol. 33, no. 2, pp. 177-202, 2010.

[25] J. Yang, R. Zhang, R. Liu et al., "Pedestrian dynamics model and simulation study based on discrete element method of particle flow," Transportation Systems Engineering and Information, vol. 16, pp. 51-57, 2016. 